Please do not remove this page

RMIT

UNIVERSITY

\title{
Microstructural and compositional analysis of strontium-doped lead zirconate titanate thin films on gold-coated silicon substrates
}

Sriram, Sharath; Bhaskaran, Madhu; Mitchell, D R G; Short, Ken; Holland, Anthony; Mitchell, Arnan

https://researchrepository.rmit.edu.au/esploro/outputs/9921863902701341/filesAndLinks?institution=61RMIT_INST\&index=null

Sriram, S., Bhaskaran, M., Mitchell, D. R. G., Short, K., Holland, A., \& Mitchell, A. (2009). Microstructural and compositional analysis of strontium-doped lead zirconate titanate thin films on gold-coated silicon substrates. Microscopy and Microanalysis, 15(1), 30-35. https://doi.org/10.1017/S1431927609090072

Published Version: https://doi.org/10.1017/S1431927609090072

Repository homepage: https://researchrepository.rmit.edu.au

(C) 2009 Microscopy Society of America.

Downloaded On 2023/04/26 18:44:42 +1000 


\title{
Microstructural and Compositional Analysis of Strontium-Doped Lead Zirconate Titanate Thin Films on Gold-Coated Silicon Substrates
}

\author{
S. Sriram, ${ }^{1, \star}$ M. Bhaskaran, ${ }^{1}$ D.R.G. Mitchell, ${ }^{2}$ K.T. Short ${ }^{2}$ A.S. Holland, ${ }^{1}$ and A. Mitchell ${ }^{1}$ \\ ${ }^{1}$ Microelectronics and Materials Technology Centre, School of Electrical and Computer Engineering, RMIT University, \\ GPO Box 2476V, Melbourne, Victoria 3001, Australia \\ ${ }^{2}$ Institute of Materials Engineering, Australian Nuclear Science and Technology Organisation (ANSTO), \\ PMB 1, Menai, New South Wales 2234, Australia
}

\begin{abstract}
This article discusses the results of transmission electron microscopy (TEM)-based characterization of strontium-doped lead zirconate titanate (PSZT) thin films. The thin films were deposited by radio frequency magnetron sputtering at $300^{\circ} \mathrm{C}$ on gold-coated silicon substrates, which used a $15 \mathrm{~nm}$ titanium adhesion layer between the $150 \mathrm{~nm}$ thick gold film and (100) silicon. The TEM analysis was carried out using a combination of high-resolution imaging, energy filtered imaging, energy dispersive X-ray (EDX) analysis, and hollow cone illumination. At the interface between the PSZT films and gold, an amorphous silicon-rich layer (about $4 \mathrm{~nm}$ thick) was observed, with the film composition remaining uniform otherwise. The films were found to be polycrystalline with a columnar structure perpendicular to the substrate. Interdiffusion between the bottom metal layers and silicon was observed and was confirmed using secondary ion mass spectrometry. This occurs due to the temperature of deposition $\left(300^{\circ} \mathrm{C}\right)$ being close to the eutectic point of gold and silicon $\left(363^{\circ} \mathrm{C}\right)$. The diffused regions in silicon were composed primarily of gold (analyzed by EDX) and were bounded by (111) silicon planes, highlighted by the triangular diffused regions observed in the two-dimensional TEM image.
\end{abstract}

Key words: strontium-doped PZT thin films, TEM, SIMS, interdiffusion

\section{INTRODUCTION}

Strontium-doped lead zirconate titanate (Sr-doped PZT or PSZT) is reputed for its relatively high piezoelectric response (Bedoya et al., 2000; Zheng et al., 2002; Sriram et al., 2007). This makes PSZT a suitable material for sensing, actuation, and transduction applications. PSZT deposited in the form of thin films can be incorporated into siliconbased devices; thereby, allowing CMOS logic and microsystem devices to coexist on the same die. For PSZT to exhibit the desired piezoelectric behavior, the films need to be of the perovskite structure and would need to be deposited on metal-coated silicon (to capitalize on the piezoelectric effect exhibited across the thickness of the film). For these reasons, PZT and doped-PZT thin films are generally deposited on platinum-coated silicon (Wasa et al., 2004; Sriram et al., 2006a). To attain the desired perovskite structure on platinum, thin film deposition needs to be carried out at temperatures in the range of $600-700^{\circ} \mathrm{C}$, or if carried out at lower temperatures, the films needed to be annealed after

Received March 30, 2008; accepted October 17, 2008

*Corresponding author. E-mail: sharath.sriram@gmail.com deposition at these temperatures (Wasa et al., 2004; Sriram et al., 2006a). To increase the range of devices in which PSZT piezoelectric thin films can be incorporated, deposition temperatures lower than the conventional $600^{\circ} \mathrm{C}$ are desirable. Previous work has identified conditions for deposition of PSZT thin films on gold at $300^{\circ} \mathrm{C}$, with the desired crystalline orientation (Bhaskaran et al., 2006). Gold was chosen as its lattice constants were similar to the major interplanar spacings in PSZT. ${ }^{\mathrm{a}, \mathrm{b}}$

This article reports on the results of transmission electron microscopy (TEM)-based studies of PSZT thin films deposited on gold-coated silicon substrates. Films were deposited for durations of 2 and $4 \mathrm{~h}$, and were about 500 and $1100 \mathrm{~nm}$ thick, respectively. The TEM analysis was carried out using a combination of energy filtered imaging, high resolution imaging, and hollow cone illumination (HCI) to observe the grain structure of the PSZT thin films. Energy dispersive X-ray (EDX) analysis and secondary ion mass

${ }^{\text {a }}$ Powder Diffraction Pattern Files, International Centre for Diffraction Data (ICDD, formerly the Joint Committee for Powder Diffraction Studies), Newtown Square, PA 19073, Card 04-0784.

${ }^{b}$ Powder Diffraction Pattern Files, International Centre for Diffraction Data (ICDD, formerly the Joint Committee for Powder Diffraction Studies), Newtown Square, PA 19073, Card 04-002-5985. 
Table 1. RF Magnetron Sputtering Conditions for PSZT.

\begin{tabular}{|c|c|}
\hline Target & $\left(\mathrm{Pb}_{0.92} \mathrm{Sr}_{0.08}\right)\left(\mathrm{Zr}_{0.65} \mathrm{Ti}_{0.35}\right) \mathrm{O}_{3}$ \\
\hline Target diameter & $100 \mathrm{~mm}$ \\
\hline RF power & $100 \mathrm{~W}$ \\
\hline Target to substrate distance & $70 \mathrm{~mm}$ \\
\hline Process gas & Ar: $\mathrm{O}_{2}(9: 1)$ \\
\hline Base pressure & $9.0 \times 10^{-6}$ Torr \\
\hline Sputtering pressure & $1.0 \times 10^{-2}$ Torr \\
\hline Substrate temperature & $300^{\circ} \mathrm{C}$ \\
\hline Sputtering duration & 2 and $4 \mathrm{~h}$ \\
\hline
\end{tabular}

spectrometry (SIMS) were carried out to study the compositional uniformity in the thin films.

\section{Experimental Details}

\section{Deposition of PSZT Thin Films}

Silicon substrates with (100) surfaces used for PSZT thin film deposition were rinsed in buffered hydrofluoric acid to remove the native oxide layer, prior to metal deposition. They were then coated with a $15 \mathrm{~nm}$ titanium adhesion layer followed by a $150 \mathrm{~nm}$ gold layer. Both metals were deposited by electron beam evaporation without substrate heating. Metal-coated samples were then rinsed in solvents (acetone and isopropyl alcohol) and deionized water. Samples were heated at $10^{\circ} \mathrm{C} / \mathrm{min}$ to the deposition temperature of $300^{\circ} \mathrm{C}$ and held at temperature during deposition. PSZT thin films were deposited by radio frequency (RF) magnetron sputtering under the conditions listed in Table 1. After deposition, samples were cooled at $5^{\circ} \mathrm{C} / \mathrm{min}$, as this improved the relative intensity of the perovskite X-ray diffraction peaks for the films (Sriram et al., 2006a).

\section{Transmission Electron Microscopy Analysis}

Cross-sectional TEM samples were prepared by mechanically polishing using a tripod polisher to create wedgeshaped specimens, with final stages of polishing performed on a $1 \mu \mathrm{m}$ diamond lapping sheet. The specimens were then ion milled to electron transparency at room temperature using $4.5 \mathrm{kV}$ argon ions incident at $5^{\circ}$ (using double beam modulation). PSZT is a ceramic oxide and exhibits the characteristics of a brittle material. This limits the TEM specimen preparation process. During ion milling for specimen preparation, silicon mills significantly faster that the PSZT thin film; this results in the entire film structure falling off, limiting the extent to which the specimens could be thinned. The analysis was carried out at an accelerating voltage of $200 \mathrm{kV}$ on a JEOL 2010F TEM equipped with a Gatan Imaging Filter (GIF2001) and an EmiSpec E Vision EDX system.
HCI was used as it excites more crystallites than conventional dark field illumination using a single diffracted beam or section of a diffraction ring. An objective aperture with a semiangle of 2.1 mrads was used, with excitation of the principal Bragg reflections in the approximate range 4-8 mrads (d-spacing 1.5-3 $\AA$ ).

\section{Secondary Ion Mass Spectrometry Analysis}

The SIMS analysis was carried out using cesium ions (Cs+) with a primary accelerating voltage of $7.5 \mathrm{keV}$ and a sampling voltage of $4.5 \mathrm{keV}$ (Cameca $5 \mathrm{f}$ dynamic SIMS instrument). Considering the insulating nature of the film, the sample was biased with an offset voltage of $+50 \mathrm{~V}$ to overcome charging effects. In view of the low concentration of strontium in the PSZT thin film samples, the two major isotopes- ${ }^{86} \mathrm{Sr}$ and ${ }^{88} \mathrm{Sr}$ - were studied to confirm the presence of strontium. The plotted data have been normalized with respect to the cesium signal measured.

\section{Results AND Discussion}

\section{Film Composition}

Figure 1 shows a low magnification view of the film. EDX analysis was carried out along the length and through the thickness of the film to detect gross variations in chemical composition due to second phases or other contaminants; none were found. The PSZT film was uniform in thickness $(\sim 470 \mathrm{~nm})$. The dark band beneath the film is the goldtitanium-silicon layer (typically $\sim 135 \mathrm{~nm}$ thick), from which prismatic ingrowths into the silicon substrate can be seen. SIMS depth profiles are indicative of average composition uniformity of the film, and the depth profile for a PSZT thin film deposited on gold-coated silicon is shown in Figure 2. Because there are no gross variations in any of the signals from the film surface to the PSZT-Au interface, it does indicate that (averaging over area of analysis) the film is compositionally uniform through its thickness. X-ray photoelectron spectroscopy data (not shown) confirmed that the final film composition matched that of the sputtering target, and related data have been previously reported (Sriram et al., 2006a). The SIMS data also highlighted some dissolution of the titanium into the gold layer, although the peak in the titanium signal corresponded to the location of the original titanium layer (between the gold layer and the silicon substrate).

Detailed imaging of this interfacial region (Fig. 3) showed an amorphous layer approximately $4 \mathrm{~nm}$ thick. Point analysis with EDX showed that this layer was rich in silicon and oxygen, most likely $\mathrm{SiO}_{2}$ formed by outward diffusion of silicon from the substrate through the metal layers. This is supported by previous studies on the fast outward diffusion of silicon through grain boundaries in gold (Sumida \& Ikeda, 1991), with this silicon oxidizing in 


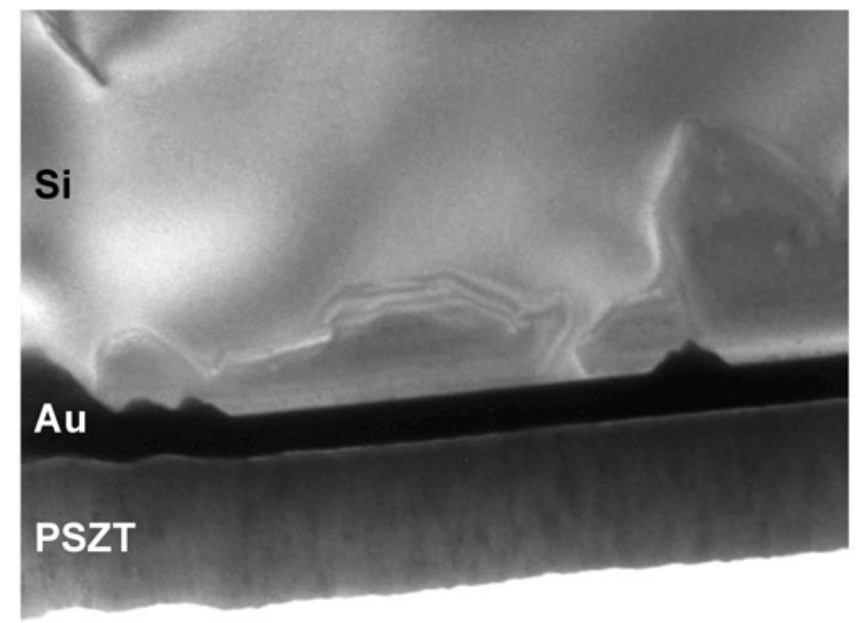

(a)

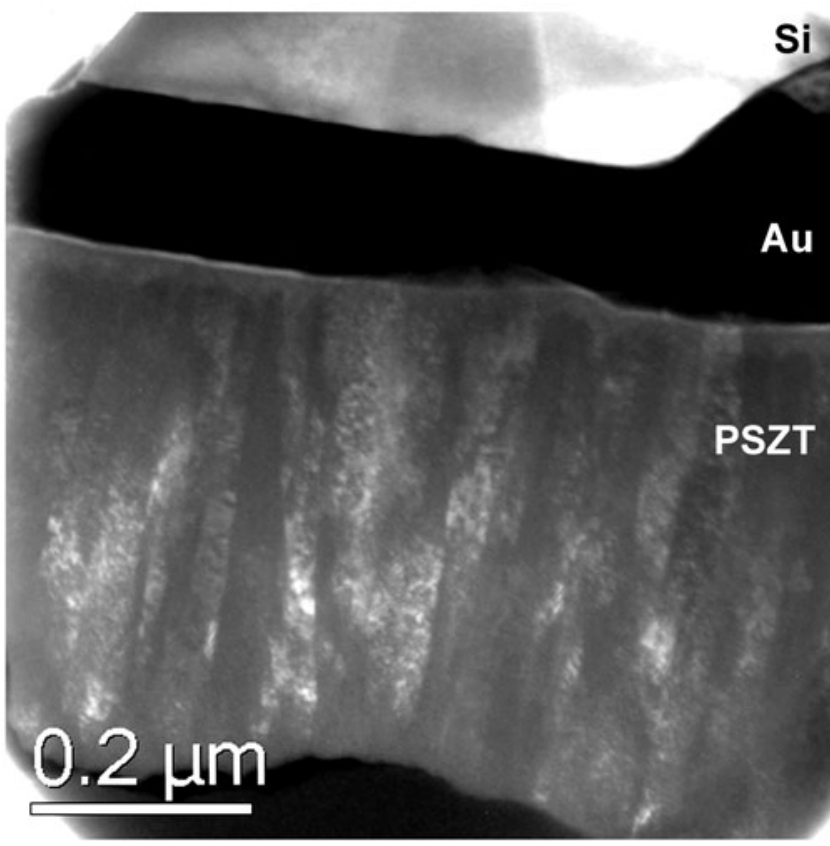

(b)

Figure 1. Cross-sectional TEM analyses of a PSZT thin film deposited for a $2 \mathrm{~h}$ sputtering duration: (a) Bright field diffraction contrast image of the $\sim 470 \mathrm{~nm}$ thick PSZT film on a $\sim 130 \mathrm{~nm}$ thick gold layer. Prismatic ingrowths of gold-silicon have formed at the interface of gold to silicon; (b) HCDF image of the PSZT film highlighting the columnar structure. Note: The outer region $(\sim 100 \mathrm{~nm})$ of the film has been removed during ion-milling.

the $10 \%$ oxygen in argon sputtering atmosphere. This is also supported by the increase in the silicon signal at this interface in the SIMS analysis (also confirming that this is not a by-product of the specimen preparation ion milling process). The region of PSZT $(\sim 25 \mathrm{~nm})$ closest to this amorphous $\mathrm{SiO}_{2}$ layer appeared to be amorphous or at best poorly crystalline, as it exhibited strong amorphous speckle (right inset of Fig. 3). Weak lattice fringe contrast was only evident only from regions greater than $30 \mathrm{~nm}$ into the PSZT layer (from the $\mathrm{SiO}_{2}$ interfacial layer, as seen in the left inset of Fig. 3).

\section{Microstructure}

The PSZT thin film exhibited a columnar microstructure (Fig. 1). These columnar grains were perpendicular to the substrate (out of plane) and were most apparent in the energy filtered (zero loss) hollow cone dark field (HCDF) image (of a 2 h PSZT deposition sample shown in Fig. 1b). Typical columnar grain widths were $30-50 \mathrm{~nm}$, and many grains appeared to span the full thickness of the film $(\sim 470 \mathrm{~nm})$. The contrast due to the crystallinity was quite weak in the $25 \mathrm{~nm}$ thick band of PSZT closest to the gold layer, confirming the amorphous or poorly crystalline nature of this region (as observed in Fig. 3). During the initial stages of deposition, the PSZT is deposited onto a substrate covered with amorphous $\mathrm{SiO}_{2}$, which affected crystallinity. Crystallization of the PSZT occurs once a certain thickness of material $(\sim 25 \mathrm{~nm})$ has been deposited. Growth stress may contribute to the onset of crystallization (Mitchell et al., 2003). X-ray diffraction analysis of these films showed that the films exhibited preferential [104] orientation (not shown here).

Increasing the deposition duration from 2 to $4 \mathrm{~h}$ increased the columnar grain width (measured from HCDF images) from $30-50$ to $\sim 90 \mathrm{~nm}$ (not shown). It also resulted in an increase in the average surface roughness $\left(R_{a}\right)$ from 7.8 to $9.5 \mathrm{~nm}$ (Sriram et al., 2006b, 2007). This was attributed to the development of marked faceting. Figure 1a shows a cross section of the relatively flat surface of the film deposited for $2 \mathrm{~h}$. The dimension of the weak faceting is similar to that of the columnar grain width $(30-50 \mathrm{~nm})$. For the film deposited for $4 \mathrm{~h}$, very pronounced prismatic faceting has occurred (Fig. 4). This is much coarser than seen at the shorter duration and reflects the grain coarsening taking place in the film in longer duration depositions. The increase in roughness is a consequence of grain coarsening, which can be attributed to the variations in growth rates as a function of crystal orientation as well as grain coalescence. At the growing surface, those grains in orientations promoting faster growth can dominate neighboring grains and eventually isolate them. With continued growth, grains may then develop prismatic faceting that causes increased surface roughening as the grain width increases (Mitchell et al., 2003). Figure 4 has lesser contrast than Figure 1a due to significantly less electron transmission. Limitations in sample preparation (as discussed in the Transmission Electron Microscopy Analysis section) did not allow us to achieve thinner specimens that preserved surface structure (which shows prismatic faceting). In regions where a portion of the film surface was milled off and the specimen was thinner (providing better contrast), columnar grain structure in the film was observed. 


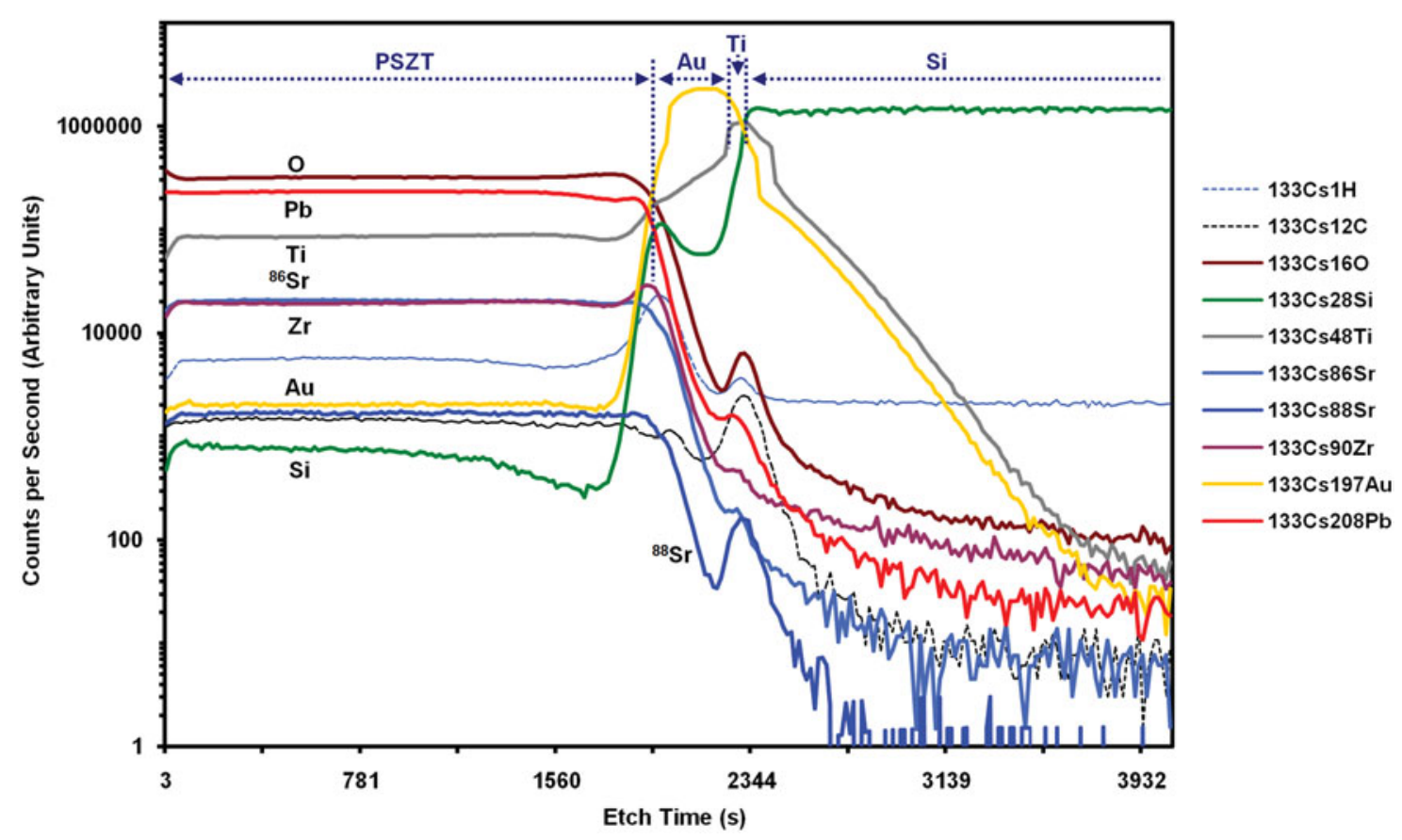

Figure 2. SIMS depth profile through the thin film.

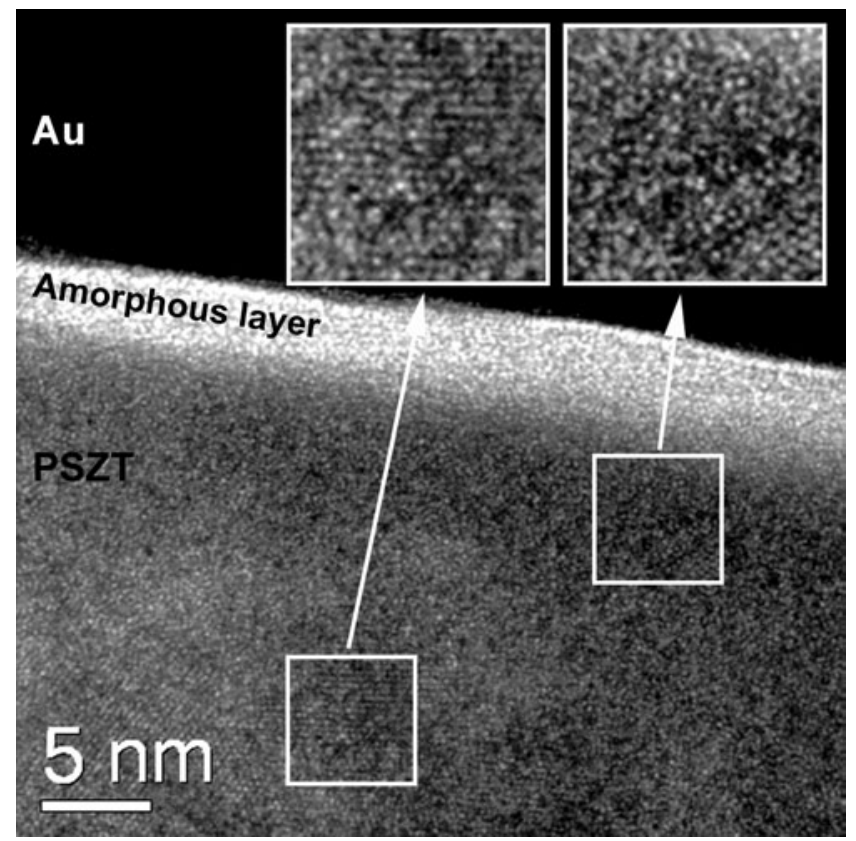

Figure 3. High-resolution energy filtered cross-sectional TEM image of the amorphous layer at the interface of PSZT and gold.

\section{Interdiffusion in the Metal Layers}

The eutectic point of gold and silicon is $363^{\circ} \mathrm{C}$, but at the deposition temperature of $300^{\circ} \mathrm{C}$ interdiffusion of gold and silicon (enabled by outward silicon diffusion through gold

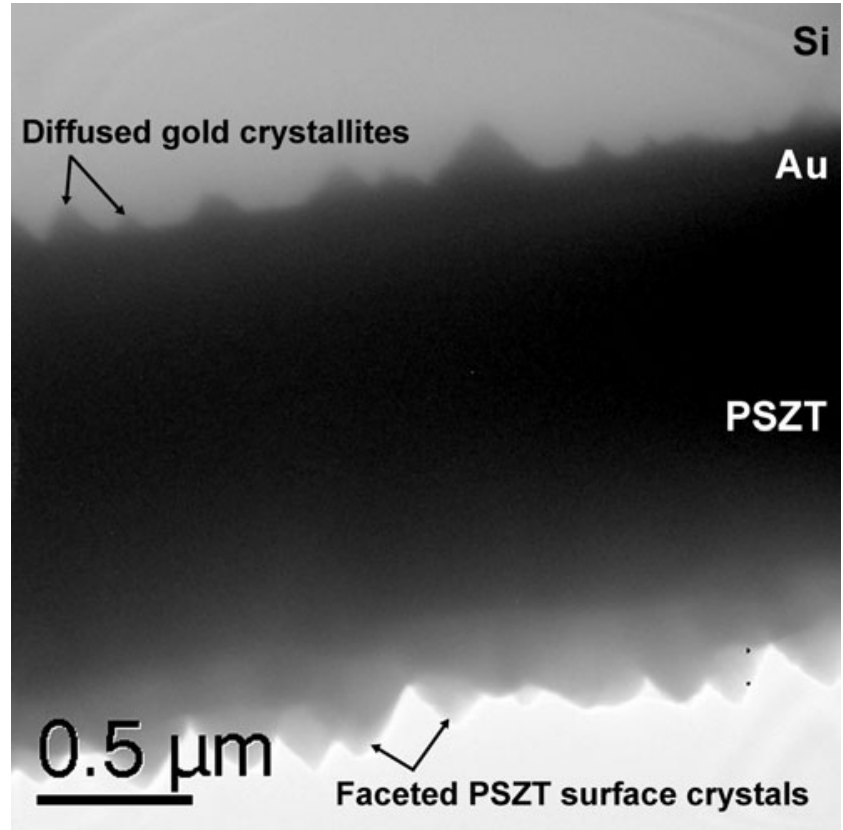

Figure 4. Cross-section energy filtered TEM image showing the highly faceted film surface with crystallites at the gold-silicon interface.

grain boundaries) through the $15 \mathrm{~nm}$ thin titanium adhesion layer has been observed. This reaction was verified using SIMS depth profiling (Fig. 2). The peak titanium concentration occurred between the gold and silicon- 


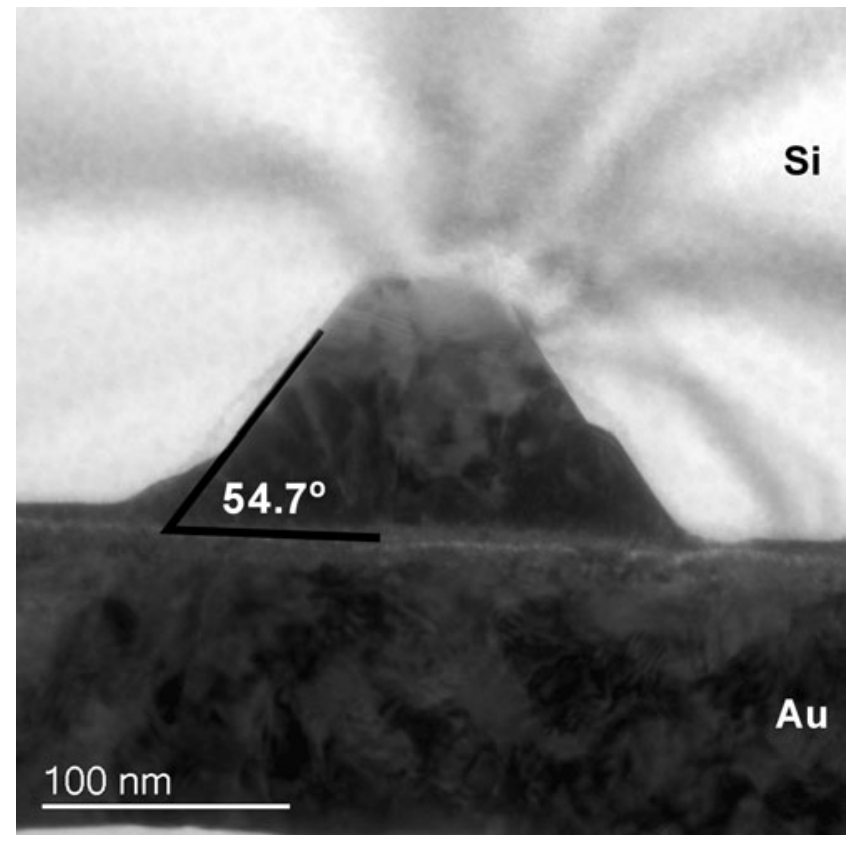

Figure 5. Cross-sectional energy filtered TEM image of a crystallite bounded by dense (111) silicon planes. The angle between the silicon (100) surface and the crystallite edge is $54.7^{\circ}$ being the silicon $(100) \wedge(111)$ interplanar angle. Note: The electron beam is parallel to silicon [110].

being the original location of the $\sim 15 \mathrm{~nm}$ thick titanium layer. On moving through the gold layer toward the PSZT (a distance of $150 \mathrm{~nm}$ ), the titanium signal showed a gradual decrease. This indicated that titanium diffused into the gold. Both titanium and gold diffusion into silicon was also apparent. The profiles for these two elements were very similar and gradually decreased over a considerable distance into the silicon. Due to the very different sputtering rates of the various phases present, it was not possible to meaningfully estimate the depth over which the gold and titanium diffused into the silicon substrate. However, the crosssectional TEM imaging provided an understanding of the origin of the SIMS profile shapes for these elements.

The gold-(titanium)-silicon reaction can be observed in the form of prismatic crystallites growing into silicon along the metal-silicon interface. This is shown in Figures 1 and 4 and in detail in Figure 5. EDX analysis confirmed that these crystallites were a reaction product of gold and siliconwith titanium also present (Fig. 6). The remnant of the original titanium layer could be seen as an $\sim 10 \mathrm{~nm}$ wide band running across the base of the crystallite in Figure 5. The crystallites were variable in size, the largest being about $200 \mathrm{~nm}$ into silicon. During SIMS depth profiling (Fig. 2), the floor of the ion milling crater would break through the main (flat) part of the metal layer into the silicon substrate, and the gold and titanium signals would be expected to drop abruptly. However, the presence of prismatic crystallites rich in gold, with traces of titanium, projecting into the

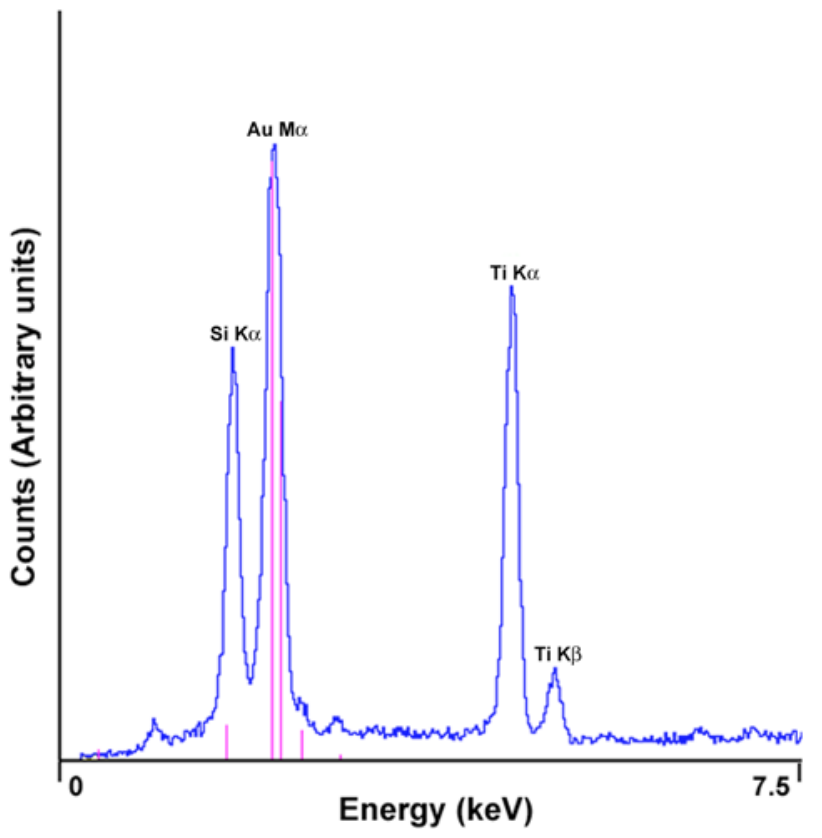

Figure 6. EDX profile of the crystallite shown in Figure 5.

silicon substrate, would cause the signal for these elements to taper off gradually as the depth profiling progressed, dropping to zero when the profile passed through the apex of the largest crystallites.

The diffusion of gold into the (100) silicon results in crystallites bounded by the dense (111) silicon planes (Fig. 5). As expected, the angle between these bounding planes and the silicon (100) surface was found to be $54.7^{\circ}$ in Figure 5, which is oriented with the electron beam parallel to silicon [110]. The resulting crystallites varied in size, and the density of such crystallites was much higher in a longer duration deposition $(4 \mathrm{~h})$ specimen. Such diffusion or dissolution of gold into silicon-based materials through titanium has been observed (Wenzel et al., 1998) and can be related to the high reactivity of titanium with silicon $\left(\mathrm{Ti}_{x} \mathrm{Si}_{y}\right.$ compounds are formed even at slightly elevated temperatures) and the thickness $(15 \mathrm{~nm})$ of the titanium layer. It has also been concluded that the titanium layer needs to have a sufficient thickness, depending on process temperature and duration, to serve as a barrier to prevent the gold-silicon reaction (Kanamori \& Sudo, 1982), rather than serve only as an adhesion layer.

\section{CONCLUSIONS}

TEM-based characterization of PSZT thin films deposited on gold-coated silicon substrates has been carried out. The PSZT thin films had a columnar grain structure. PSZT films exhibited a uniform composition through their thickness. A silicon-rich amorphous layer was formed at the interface of 
the PSZT thin films and gold, and was most likely to be silicon dioxide. Prismatic crystallites of a gold-silicon compound developed at the interface of the metal layers and the silicon substrate. This was caused by gold diffusing through the underlying titanium metal layer, the crystallites being bounded by the dense (111) silicon planes.

\section{ACKNOWLEDGMENTS}

The authors acknowledge support for this project from the Australian Institute of Nuclear Science and Engineering and The CASS Foundation. The authors also thank Armand Atanacio for his assistance with the SIMS analysis.

\section{REFERENCES}

Bedoya, C., Muller, Ch., Baudour, J.-L., Madigou, V., Anne, M. \& Roubin, M. (2000). Sr-doped $\mathrm{PbZr}_{1-x} \mathrm{Ti}_{x} \mathrm{O}_{3}$ ceramic: Structural study and field-induced reorientation of ferroelectric domains. Mater Sci Eng B 75, 43-52.

Bhaskaran, M., Sriram, S. \& Holland, A.S. (2006). RF magnetron sputtered perovskite-oriented PSZT thin films on gold for piezoelectric and ferroelectric transducers. Electron Lett 42, 244-245.

Kanamori, S. \& Sudo, H. (1982). Effects of titanium layer as diffusion barrier in $\mathrm{Ti} / \mathrm{Pt} / \mathrm{Au}$ beam lead metallization on poly- silicon. IEEE Trans Comp Hybrids Manuf Tech CHMT-5, 318-321.

Mitchell, D.R.G., Attard, D.J. \& Triani, G. (2003). Transmission electron microscopy studies of atomic layer deposition $\mathrm{TiO}_{2}$ films grown on silicon. Thin Solid Films 441, 885-895.

Sriram, S., Bhaskaran, M. \& Holland, A.S. (2006a). The effect of post-deposition cooling rate on the orientation of piezoelectric $\left(\mathrm{Pb}_{0.92} \mathrm{Sr}_{0.08}\right)\left(\mathrm{Zr}_{0.65} \mathrm{Ti}_{0.35}\right) \mathrm{O}_{3}$ thin films deposited by $\mathrm{RF}$ magnetron sputtering. Semiconductor Sci Tech 21, 1236-1243.

Sriram, S., Bhaskaran, M. \& Holland, A.S. (2006b). Surface morphology and stress analysis of piezoelectric strontiumdoped lead zirconate titanate thin films. In Micro- and Nanotechnology: Materials, Processes, Packaging, and Systems III, Chiao, J.-C., Dzurak, A.S., Jagadish, C. \& Thiel, D.V. (Eds.), p. 64150J. Proceedings of the SPIE, Vol. 6415.

Sriram, S., Bhaskaran, M., Holland, A.S., Short, K.T. \& LaTELLA, B.A. (2007). Measurement of high piezoelectric response of strontium-doped lead zirconate titanate thin films using a nanoindenter. J Appl Phys 101, 104910.

Sumida, N. \& Ikeda, K. (1991). Cross-sectional observations of gold-silicon reaction on silicon substrate in situ in the highvoltage electron microscope. Ultramicroscopy 39, 313-320.

Wasa, K., Kiтabatake, M. \& Adachi, H. (2004). Thin Film Materials Technology: Sputtering of Compound Materials. Heidelberg, Germany: Springer-Verlag GmbH \& Co. KG.

Wenzel, R., Goesmann, F. \& Schmid-Fetzer, R. (1998). Diffusion barriers in gold-metallized titanium-based contact structures on SiC. J Mater Sci: Mater Electron 9, 109-113.

Zheng, H., Reaney, I.M., Lee, W.E., Jones, N. \& Thomas, H. (2002). Surface decomposition of strontium-doped soft $\mathrm{PbZrO}_{3}-$ $\mathrm{PbTiO}_{3}$. J Am Ceram Soc 85, 207-212. 